\title{
Professional Development Opportunities
}

by Leslie A. Engelson

\section{SOFTWARE \& AUTOMATION}

\section{Regular Expressions}

This introductory course teaches the syntax and construction of regular expressions. Students will follow patterns to construct examples.

April 21 \& 23, 2020

$\$ 140-\$ 220$

\section{Excel for Technical Services: Introductory and Intermediate}

A two-part series that will provide attendees with the skills to create and use filters, create a pivot table, use formulas, import data from one spreadsheet into another, use conditional formatting, and split cells that contain multiple values.

April 22 \& May 20, 2020

\$43-\$206 each

Testing for Accessibility: Free Tools to Assess the Accessibility of Online Resources

Students will learn to identify the standards and best practices used to assess accessibility of webbased resources, apply them in the decision-making process, and use tools to test accessibility.

April 23, 2020

$\$ 75-\$ 100$

\section{Programming with Python and PyMARC}

Learn the basics of the Python language to automate workflows and metadata management processes. Understand PyMARC and its uses in libraries.

May $19 \& 21,2020$

$\$ 140-\$ 220$

\section{MANAGEMENT}

\section{Know \& Go: Increase Your Productivity with Time Management}

Learn how to assess the value of your tasks, set goals, and use time management principles to increase productivity.

May 4, 2020

$\$ 0-\$ 35$ 


\section{Make That Decision: Strategies for Becoming a Better Decision Maker}

Several decision-making strategies such as risk-taking perspective, slow decision-making, avoiding paralysis, and collaborative decision-making will be explored. Participants will also learn behavioral aspects of decision-making which will increase the odds for better outcomes and increase comfort with risk.

August 12, 2020

$\$ 100-\$ 200$

Demonstrating the Return on Investment: The Library's Role and Contribution

Use metrics to demonstrate that your library is a cost-effective and impactful part of the academic program. Participants will learn how well library initiatives are aligned with institutional goals and how assessment exercises are critical for effectively presenting the library's value.

September 9, 2020

$\$ 0-\$ 135$

\section{RDA}

\section{LRM: A New Foundation for RDA and the RDA Toolkit}

Attendees will become familiar with the Library Reference Model (LRM) and changes in terminologies, compare mappings between FRBR, FRAD, and FRSAD to LRM, and review approaches to cataloging following LRM.

June 1-28, 2020

$\$ 175$ 